\title{
The Shape of the Hyphal Tips of Fungi
}

\author{
By ARTHUR L. KOCH \\ Department of Biology, Indiana University, Bloomington, Indiana 47405, U.S.A.
}

(Received 22 April 1981; revised 24 July 1981)

\begin{abstract}
A growing hyphal tip is analogous to a molten glass bubble blown under special conditions. These conditions are that the pressure is constant throughout the tip and that the surface tension is very low at the apex and increases towards infinity with distance from the tip. The biological correlate for low surface tension at the apex of the hypha is that enzymic activity and rate of fusion of vesicles are very high in the immediate vicinity of the apex. A formula is developed to allow the calculation of the rate of synthesis at a point on the tip from its distance from the axis and the slope of the tip at that point.
\end{abstract}

\section{INTRODUCTION}

A general model for bacterial wall growth has been proposed and specifically applied to Streptococcus faecium (Koch et al., 1981). The model is simply that tension in the wall in regions where wall enlargement is taking place determines the shape of the organism. More recently, we have extended it to the nearly spherical cocci and to rod-shaped bacteria (Koch et al., 1982). In this note, the model is extended in a different way to cover the growing hyphal tips of fungi. The possibility is left open that this case may also apply to the hyphal tips of filamentous actinomycetes. This work is an extension of that of Thompson (1942), Trinci \& Saunders (1977) and Saunders \& Trinci (1979).

A growing hyphal tip is a projectile propelled through the environment (sometimes through quite viscous and resistant media) by growth due to metabolic processes in the entire mycelium. The hyphal walls elongate essentially only at the tips, although net macromolecular synthesis takes place in much of the mycelial mass. The products are packaged in vesicles (spitzenkörper) which fuse with the apex and lead to enlargement of the wall (see Gooday \& Trinci, 1980). The problem of determining the shape of hyphal tips within the framework of our general model is different from calculating the shapes of the poles of bacteria. The shape of the latter is determined by the wall growth process leading to cell division. As we noted before, the formation of septal or cross-wall material occurs under a different set of conditions from those which apply after this wall material is externalized. The degree to which septal material is reworked and combined with new material to form external wall greatly affects the shape of the nascent poles. In fact, if the properties of the process leading to wall enlargement are constant throughout the cycle, at the final instant of cell division, previously formed septal material has to be the source of all of the strain-bearing wall of the tip. Moreover, the elongation of hyphae is different from the elongation of rod-shaped bacteria; the latter have caps or poles on both ends while the former only has a reference structure on one end. What is special about tip growth is that the growth leads to a shape identical to that at a previous instant of time, but spacially displaced. This means that at a particular point in the elongation zone where the radius is $r$ and the slope is $S(=\mathrm{d} r / \mathrm{d} z)$, growth is such that at a different but corresponding point an instant later the radius is $r$ and the slope is again $S$. This is shown diagrammatically in Fig. 1. 


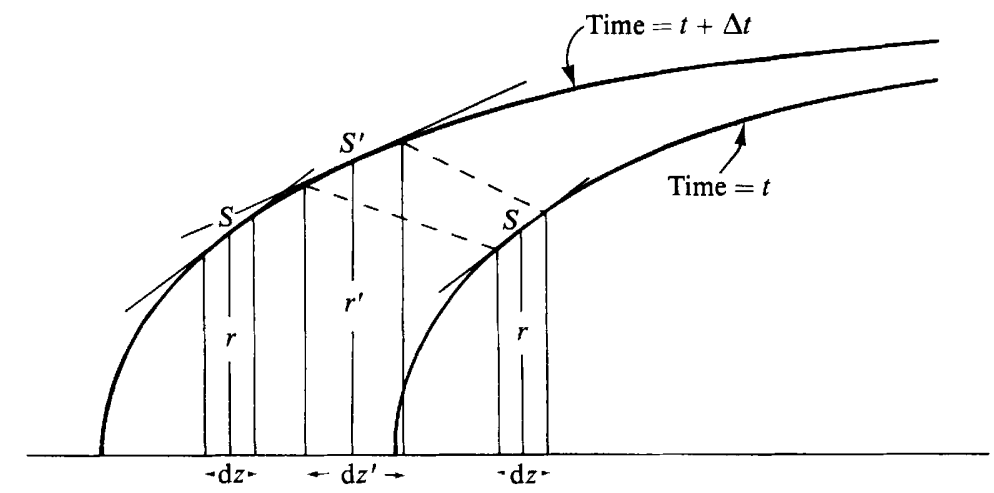

Fig. 1. The shape of a growing hyphal tip is shown at time $t$ and at a later time, $t+\Delta t$. For time $t$, a hypothetical section is labelled with its width, $\mathrm{d} z$, its cylindrical radius, $r$, and the slope of its surface, $S$. The same quantities with primes are used to label the same region now enlarged and displaced at time $t$ $+\Delta t$. If the width of the section increases proportionally to time, we may set $\mathrm{d} z^{\prime}=(1+a \Delta t) \mathrm{d} z$, where $a$ is the rate of axial elongation. The increase in $T \mathrm{~d} A$ is:

$$
2 \pi T\left\{\left(S^{\prime 2}+1\right)^{1 / 2}\left(r^{\prime}\right)(1+a \Delta t)-\left(S^{2}+1\right)^{1 / 2} r\right\} \mathrm{d} z
$$

The increase in $P \mathrm{~d} v$ is:

$$
\pi P\left\{r^{2}(1+a \Delta T)-r^{2}\right\} \mathrm{d} z
$$

After equating these work terms and cancelling $\pi \mathrm{d} z$, we obtain:

$$
2 T\left\{\left(S^{\prime 2}+1\right)^{1 / 2}\left(r^{\prime}\right)(1+a \Delta t)-\left(S^{2}+1\right)^{1 / 2} r\right\}=P\left\{r^{\prime 2}(1+a \Delta t)-r^{2}\right\}
$$

In the limit where $\Delta t \rightarrow 0$, then $r^{\prime} \rightarrow r$ and $S^{\prime} \rightarrow S$, and this relationship simplifies to:

$$
\begin{array}{cc} 
& 2 T\left(S^{2}+1\right)^{1 / 2} r a \Delta t=\operatorname{Pr}^{2} a \Delta t \\
\text { or } & \left(S^{2}+1\right)^{1 / 2}=\operatorname{Pr} / 2 T
\end{array}
$$

which, on squaring, rearranging, and taking a square root yields equation (3).

\section{THEORY}

On the assumption that hyphal growth is slow enough so that wall extension is essentially an equilibrium process, the problem becomes tractable because then the energy of the pressure-volume $(P \mathrm{~d} v)$ work of protoplasm expansion must equal the tension-surface area $(T \mathrm{~d} A)$ work:

$$
P \mathrm{~d} v=T \mathrm{~d} A
$$

For most of the cases so far considered (Koch et al., 1981, 1982) not only $P$, but $T$ as well, appears to be constant throughout most of the growth process. For the case of the growing hyphal tip where there is no formation of septal material, it is necessary that $T$ be a variable quantity. It has to be very small at the growing tip and become large in distal regions of the hyphae where there is to be little further growth. This statement is consistent with the fact that vesicles fuse with the wall almost exclusively at the apex. The reciprocal of $T$ may well be equated to the metabolic activity augmenting the wall, since $T$ is a measure of how easily the surface can be enlarged. The relationship of $T$ to distance along the axis of growth is readily derived from the shape of the tip. The formula can be derived using the methods of the calculus by combining equation (1) with the quantities defined in the legend to Fig. 1, as is done in the legend to Fig. 1. However, we can also grasp the solution directly by appealing to the simpler mathematical relationship based on an equation developed for a different case the externalization of septal material as pole formation takes place (Koch et al., 1981). The relationship derived there was:

$$
\left(4-\delta^{2}\right) S^{2}+2 \delta(P / T) r S+4-(P r / T)^{2}=0
$$




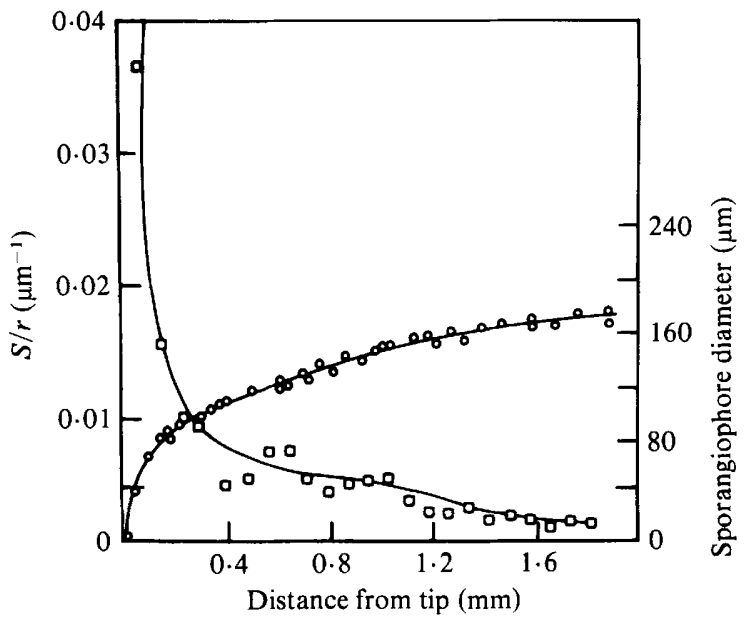

Fig. 2. $S / r$ for the Stage I sporangiophore of Phycomyces blakesleeanus. The diameter measurements of Castle (1958) on the growing sporangiophore are shown (O). Data were reconstructed from his Fig. $2(\mathrm{~d} z / \mathrm{d} t)$ and his Fig. $5(\mathrm{~d} r / r \mathrm{~d} t)$; the quotient of these results yielded $\mathrm{d} r / r \mathrm{~d} z=S / r$. The $S / r$ values $(\square)$ have been plotted against axial distance, $z$.

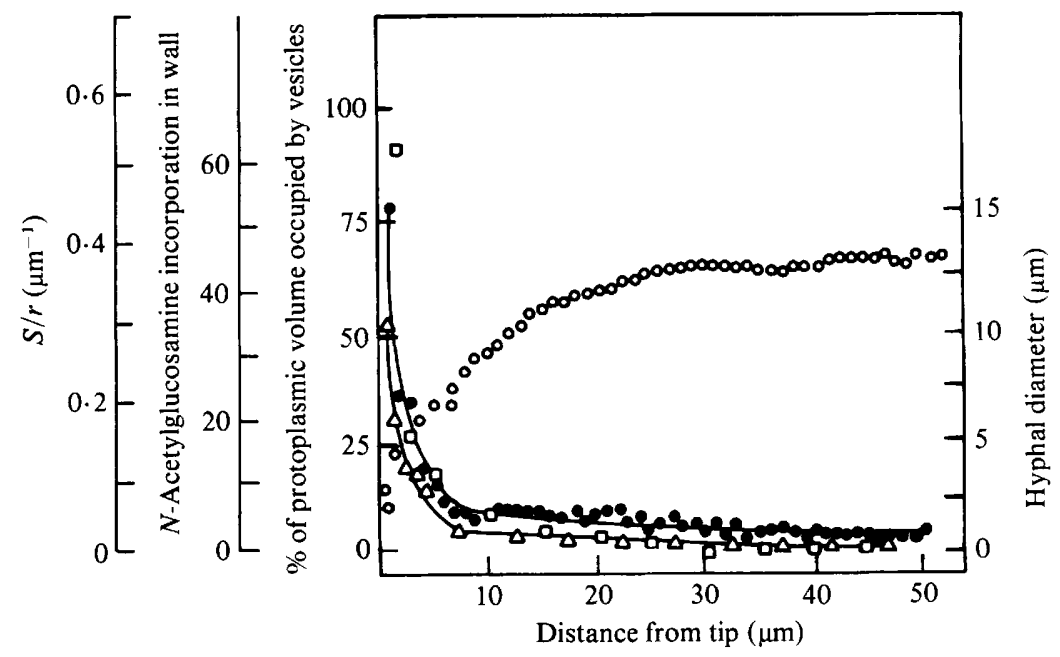

Fig. 3. $S / r$ for the hyphal tip of Neurospora crassa ( $\square$ ). Data were computed from Fig. 11 of Collinge \& Trinci (1974). They have been superimposed on Fig. 3 of Gooday \& Trinci (1980) which shows the specific rate of incorporation of $N$ - $\left[{ }^{3} \mathrm{H}\right]$ acetyl-D-glucosamine $(\triangle)$, the percentage of the protoplasmic volume occupied by vesicles $(O)$ and hyphal diameter $(O)$.

All symbols for this equation have been defined except $\delta$; this is the thickness of the septum relative to the thickness of the wall. For the present application, there is no septum and therefore $\delta=0$. Consequently, the equation simplifies and can be solved for $S$ to yield:

$$
S=\left\{(\operatorname{Pr} / 2 T)^{2}-1\right\}^{1 / 2}
$$

which approaches

$$
S=P r / 2 T
$$

when $\operatorname{Pr} / T$ is much greater than 2 . 
Of course, the pressure, $P$, must be constant throughout the hyphal tip at a single instant of time. $T$ appears to be constant in both the narrow and diffuse growth zones of Gram-positive bacteria (Koch et al., 1981, 1982). However, it need not be constant over the entire growing wall and would depend on the metabolic properties of the wall-biosynthetic system throughout the entire growing area. From the measured shape of a hyphal tip, $S$ and $r$ at every point can be obtained and the dependency of $T$ can be calculated as a function of the axial distance, $z$, from the tip. This is easily done as suggested by the following rearrangement of equation (4):

$$
S / r=(\mathrm{d} r / \mathrm{d} z) / r=P / 2 T
$$

Since it is more to the point to calculate the reciprocal of the surface tension because $1 / T$ would be expected to be proportional to the concentration of the enzyme (or enzyme system) limiting the rate of expansion of the wall, we plot $S / r$ and assume that this is proportional to wall growth activity, i.e. we assume that $P$ is constant. Figures 2 and 3 show examples calculated from the literature where measurements of the shapes of the tips of growing hyphae of different fungi were presented. Also shown in Fig. 3 is the experimentally determined rate of wall increase estimated from the pulse incorporation of precursors by autoradiography. The agreement offers support for the validity of this approach.

\section{DIS CUSSION}

This simple analysis has made approximations at three points in its derivation, all of which I believe are minor. The derivation given in the text assumed that growth takes place in a very narrow zone. The more rigorous derivation given in the legend to Fig. 1 depends alternatively on the assumption of a steady state. The fact that the same result was obtained in two different ways suggests that it is generally valid. A still more rigorous derivation is being sought. Secondly, $S / r$ has been assumed to be proportional to $P / T$, instead of a more complex relationship based on equation (3). An absolute measurement of $T$ at some location on a growing hyphal tip would suffice to permit the exact computation of $1 / T$ at all points on the tip and allow the use of equation (3) instead of equation (5). However, this approximation should only give significant error in the regions well behind the tip where the radius is only slowly expanding. There it will lead to a slight underestimate of $1 / T$. Thirdly, as mentioned above, the hydrostatic pressure has been assumed to be constant in both space and time.

The model proposed here is not inconsistent with the previous ideas of Bartnicki-Garcia (1973), or the elegant electron microscope studies of Grove \& Bracker (1970). It is simply the statement that a tubular structure with a particular shape of tip can be constructed if new material is added mainly at the apex in an amount proportional to $S / r$ and that some additional mechanisms maintain the thickness of the wall constant at least as long as that wall has plastic properties. The value of this paper lies in the degree to which it allows the detailed distribution of the sites of wall addition to be predicted. If in further work this prediction holds, then a search should be initiated to find out how a vesicle fusion mechanism of apical growth responds sensitively to protoplasm growth. When the hydrostatic pressure tends to rise as macromolecules are formed and as materials enter the mycelium, wall growth must occur in a coordinated way in order to maintain constant intracellular hydrostatic pressure. A search should also be made for the mechanism by which vesicles can pass through the cytoplasmic membrane, fuse with the appropriate level of the wall and quickly allow the wall to enlarge in a way that leaves its thickness unchanged.

\section{REFERENCES}

BARTNICKI-GARCIA, S. (1973). Fundamental aspects of hyphal morphogenesis. Symposia of the Society for General Microbiology 23, 245-267.
CASTle, E. S. (1958). The topography of tip growth in a plant cell. Journal of General Physiology 41, 913-926. 
Collinge, A. J. \& Trinci, A. P. J. (1974). Hyphal tips of wild-type and spreading colonial mutants of Neurospora crassa. Archives of Microbiology 99, 353-368.

GoodAy, G. W. \& Trincl, A. P. J. (1980). Wall structure and biosynthesis in fungi. Symposia of the Society for General Microbiology 30, 207-251.

Grove, S. N. \& Bracker, C. E. (1970). Protoplasmic organization of hyphal tips among fungi: vesicles and spitzenkörper. Journal of Bacteriology 104, 989-1009.

Koch, A. L., Higgins, M. L. \& Doyle, R. J. (1981). Surface tension-like forces determine bacterial shapes: Streptococcus faecium. Journal of General Microbiology 123, 151-161.
Koch, A. L., Higgins, M. L. \& Doyle, R. J. (1982). The role of surface stress in the morphology of microbes. Journal of General Microbiology 128 , 927-945.

Saunders, P. T. \& Trinci, A. P. J. (1979). Determination of tip shape in fungal hyphae. Journal of General Microbiology 110, 469-473.

ThOMPSON, D'ARCY, W. (1942). On Growth and Form, 2nd edn, pp. 351-384. Cambridge: Cambridge University Press.

Trinci, A. P. J. \& SAunders, P. T. (1977). Tip growth of fungal hyphae. Journal of General Microbiology $103,243-248$. 